\title{
Grouping Based Dynamic Framed Slotted ALOHA for Tag Anti-Collision Protocol in the Mobile RFID Systems
}

\author{
Xiaowu Li ${ }^{1,2}$ and Quanyuan Feng ${ }^{1}$ \\ ${ }^{1}$ School of Information Science and Technology, Southwest Jiaotong University, Chengdu 610031, Sichuan, China \\ ${ }^{2}$ School of Information and Technology, Kunming University, Kunming 650214, Yunnan, China
}

Received: 7 Oct. 2012; Revised: 3 Feb. 2013; Accepted: 6 Feb. 2013

Published online: 1 January 2013

\begin{abstract}
Dynamic framed slotted ALOHA (DFSA) protocol has been widely used in many RFID systems. According to the information from last frame (e.g. the number of idle, success and collision slots), DFSA dynamically adjusts the next frame size so as to reach higher identification efficiency. The process is known as tag estimation of DFSA. Similar to most existing tag anti-collision protocols, The DFSA initially aims at tag identification in static environments where all tags keep still during the tag identification process. However, in many real scenarios (called mobile RFID systems), tags usually move along a fixed path in the reader coverage area. That is, tags enter and leave the area continuously. This easily leads to serious errors of tag estimation because the tag estimation of almost existing DFSA variants do not consider new arrival tags. The errors in turn cause very low tag identification rate and eventually make some tag leave the area unidentified. In this paper, we propose a grouping based dynamic framed slotted ALOHA protocol (GBDFSA) which is very suitable for mobile RFID systems. Through tag grouping in their arrival time order, the tag estimation algorithm in GBDFSA can eliminate the influence of new arrival tags. Simulation results show that GBDFSA can significantly reduce the numbers of lost tags in mobile RFID systems. The idea of the paper is used in various existing DFSA variants so as to make these protocols adapt to mobile RFID systems.
\end{abstract}

Keywords: Anti-collision protocol, dynamic framed slotted ALOHA (DFSA), first come first served (FCFS), mobile RFID systems, tag loss ratio (TLR)

\section{Introduction}

Radio Frequency Identification (RFID) is increasingly used in many automated identification systems $[1,2,3,4]$. In the RFID systems, all the tags in the reader coverage area communicate with the reader via a shared communication channel. If more than one tag sends its information simultaneously, a collision occurs and reader can not identify them, which is called tag collision problem.

So far, a great number of collision resolution protocols $[5,6,7,8,9]$ have been proposed for static scenarios where all tags keep still during the tag identification process. Among them, many DFSA variants are widely applied to RFID systems since almost all DFSA protocols have better performance than FSA ones. DFSA protocols are characterized by dynamic frame size adjustment according to the number of idle, success or collision slots in last frame, which can improve throughput $[10,11,12,13]$. For example, Schoute in [11] proposed a tag estimation

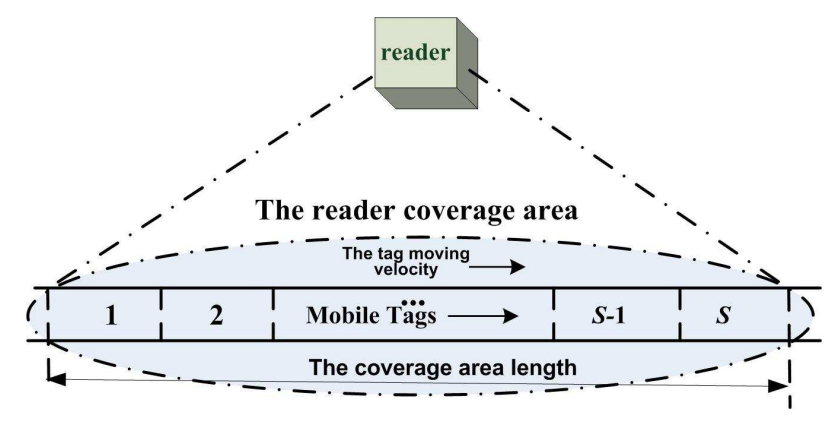

Figure 1 The mobile RFID system diagram.

method, in which the frame size is set to $2.39 C$, where $C$ is the number of collision slots in the last frame. Compared with ordinary frame slotted ALOHA (FSA) [5], Schoute's

\footnotetext{
*Corresponding author e-mail: fengquanyuan@swjtu.edu.cn; Xiaowu Li: e-mail: 1xwlxw66@ 126.com
} 


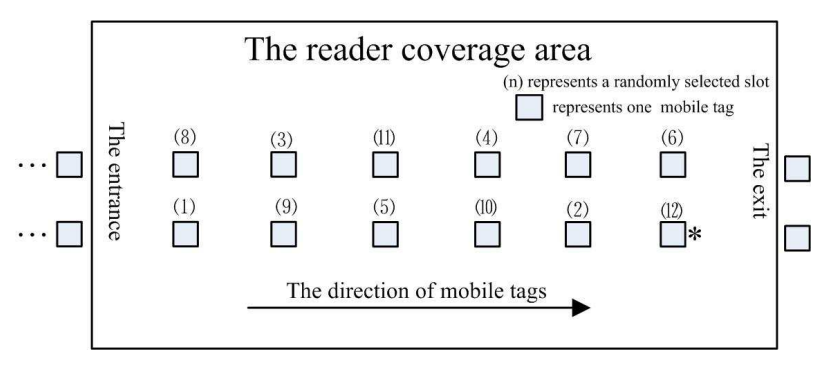

Figure 2 Random later identification phenomenon.

method improves the system performance in static scenarios.

However, in many practical applications, tags usually move along a fixed path in the reader coverage area, known as mobile RFID systems $[14,15,16,17]$. In the scenarios, tags stay the reader coverage area only for a certain time (sojourn time). Fig. 1 shows the mobile RFID system diagram and illustrates some parameters: the reader coverage area length, the tag moving velocity (assumed constant) and tag sojourn time $S$ (given in slots) which is a quotient between the reader coverage area length and the time interval for one slot. Mobile RFID systems shown in Fig. 1 are our research scenarios.

In the mobile RFID systems, since the multiple tag identification based on a shared wireless channel is random, tags entering the coverage area earlier may be identified later, which is named the random later identification (RLI) phenomenon in this paper. An example given in Fig. 2 illustrates the phenomenon. In this figure, all tags in the coverage area contend the shared wireless channel together. Each tag randomly selects a slot to communicate with the reader. For example, the tag with asterisk, which enters the coverage area earlier than other ones, is closer to the exit of the coverage area than other tags, but selects the latter slot (slot 12) for communication with the reader. So, it may leave the coverage area unidentified. Such unidentified tag is called lost tag. According to the analysis, we believe that the RLI phenomenon an important reason for tag loss.

With various existing DFSA variants, there still is another reason that results in tag loss. It is well known that DFSA protocols dynamically adjust the fame size by tag estimation algorithms. These algorithms only consider the number of successful slots, idle slots, and collision slots from the previous frame. However, in mobile RFID systems, large many errors of the tag estimation will occur inevitable since new arrival tags are not considered. This can also result in tag loss eventually.

How to decrease the number of lost tags is the critical research issue in mobile RFID applications. Therefore, we rate tag loss ratio (TLR) as a critical performance metric, which is defined as the quotient between the number of lost tags and the total number of tags entering the coverage area $[16,17]$. TLR is related to other many system performance metrics, such as throughput, system efficiency, the error rate of tag estimation and the identification delay [5]. For example, if the error rate of tag estimation of protocol A is higher than protocol $\mathrm{B}$, then the identification delay and TLR of protocol A are usually usually higher than protocol B. Compared with other performance metrics, the users of RFID systems put more emphasis on TLR because tag loss is often unpermitted for most RFID applications. So, we only focus on the performance metric TLR in the paper.

Until now, some researches on mobile RFID systems have been done $[14,15,16,17]$. [16,17] focused on TLR computation of frame slotted ALOHA protocol (FSA) by Markov model or dynamic systems model.

In $[14,15]$, authors focused on single tag set passing the reader coverage area in a limited time where no more tag sets enter the coverage area until the previous one has left. Obviously, the scenario is different from our research scenario.

In [18], Sarangan offered a framework which reduce the tag reading time by using bitmaps. The framework can improve to some extent the system performance of mobile RFID systems. However, the method also can not solve RLI problem effectively.

In this paper, we present a grouping based dynamic framed slotted ALOHA (GBDFSA) protocol. The characteristics of the protocol are: (1) The reader can group tags in the coverage area in their arrival order, which avoids influence of new arrival tags on tag quantity estimation. (2) The reader can further identify tag groups in their arrival order one by one, which indicates that GBDFSA can overcome RLI phenomenon and makes tag identification in mobile RFID systems possess the attribute of first come first served (FCFS).

The remainder of this paper is organized as follows. In Section 2, we propose the grouping based dynamic framed slotted ALOHA protocol (GBDFSA). Section 3 provides the simulation results. Finally, section 4 concludes.

\section{The Proposed Protocol}

The rules of GBDFSA protocol can be summarized as follow:

(1) The tags in the reader coverage area are grouped in their arrival order every $F G$ slots. Obtained groups are called time groups and each time group is assigned a time group sequence number $(T G S N)$. This process is called tag sequencing process. We can derive that tags arriving in the same time interval possess the same TGSN and tags arriving in different time interval possess different TGSNs. Notice that $F G$ can be known as the period of tag grouping. For the special case of static scenarios, all tags' TGSNs are the same because they enter the coverage area in the same time interval. In Fig. 3, tags in the reader coverage area are divided three groups.

(2) Outside of grouping operation hour, the reader further identifies time groups in TGSNs order one by one. This process is called further identification process. Notice 


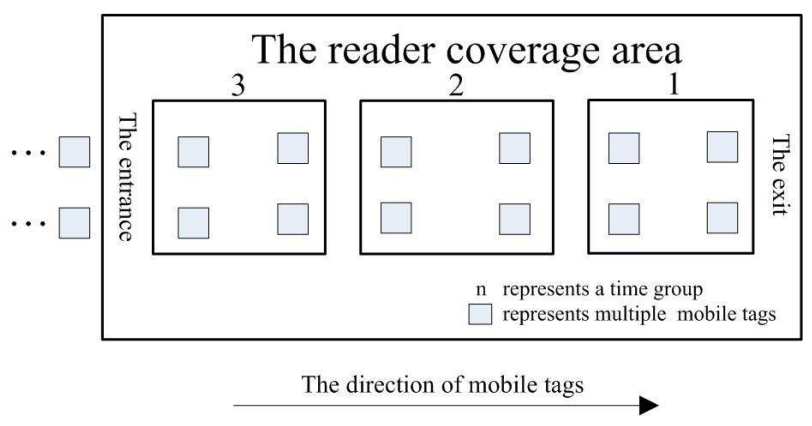

Figure 3 The tags grouped in their arrival time order.

that the earliest time group is first identified. For example, time group 1 in Fig. 3 is first identified since it entered the coverage area first. In the further identification process, we use Schoute' strategy [11] to identify time groups for simplicity, which can refer to Section 1 . Of course we also select other DFSA variants to further identify time groups. The further tag identification can be summarized as follows: The reader gathers the number of collision slots $(C)$ from the current frame. The frame size in next frame will be set to $2.39 C$, where $C$ is the number of collision slots. Notice also that the reader can group new arrival tags every $F G$ slots during the further identification process. The reason for the operation is to avoid the phenomenon of not sequencing tags for a long time when a time group contains too many tags. So, by adjusting the value of $F G$, the frequency of grouping tags in the protocol is adjustable.

In this way, GBDFSA protocol can group tags in their arrival order and each time group possesses a different $T G S N$. On this basis, the reader identifies the tag groups (time groups) in their TGSNs order. These operations eliminate influence of new arrival tags on tag estimation and resolves RLI problem. The solution to RLI phenomenon in turn means that the proposed protocol can convert random access of tags into sequential access of tag groups, that is, the protocol can made tag identification possess the attribute of FCFS.

\section{Simulation and Results}

Before we evaluate GBDFSA, we first offer a simplified mobile RFID experiment model. The reason for this is that TLR can be derived by mathematical model only for FSA and CSMA so far [16,17]. Our model comprises 3 groups of mobile tags, as shown in Fig. 4 where $T 1$ denotes the arriving interval between the 1st and 2nd groups of tags, $T 2$ denotes the arriving interval between the 2 nd and 3 rd groups of tags, $N 1, N 2$ and $N 3$ respectively denote the number of tags in the 1st, 2nd and 3rd groups of tags, $S$ denotes the tag sojourn time. Notice that in the paper, all parameters related to time such as $T 1, T 2, S$ and $F G$ are measured in slot. Parameters $S$ and $F G$ have been defined

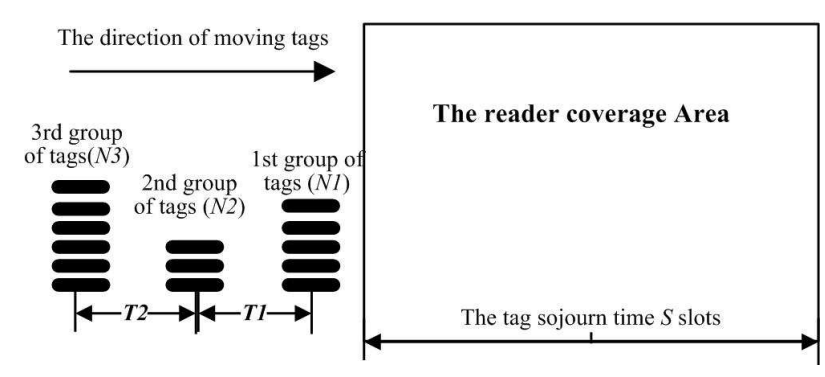

Figure 4 Simplified mobile RFID experiment model.

in section 1 and 2 . The reasons for using the simplified mobile RFID experiment model are the following:

(1) The model is a basic cell of mobile RFID systems. The behavior of the 3 groups of mobile tags in the coverage area can reflect to some extent that of real mobile RFID scenarios.

(2) As stated previously, there are no good mathematical methods that can compute the TLR of various anticollision protocols in the mobile RFID systems so far.

(3) The proposed experiment model can be very convenient to vary each experiment parameter, which help to observe the behavior of various anti-collision protocols from different perspectives.

(4) Through the model, we can compute the TLR of various anti-collision protocols conveniently, as shown in the following.

Now, we evaluate the TLR of GBDFSA and dynamic frame slotted ALOHA (DFSA) protocol [11] which is similar to GBDFSA protocol except that tags in [11] can be not grouped. We consider two protocols with early end and muting feature [6]. The term early end indicates that if no transmission is detected at the beginning of a slot, the reader closes the slot early. In our simulation, an idle slot or a slot by occupied by lost tags costs 0.1 times of a successful slot. The term Muting means that identified tags are acknowledged by the reader and withdraw themselves from contention in subsequent identification cycles. Our simulation based on Monte Carlo technique and the simplified mobile RFID experiment model.

In the Fig. 5 (a), we analyze the relationship between TLR and the tag density by changing $N 2$. Related parameters in the experiment are: $N 1=60, N 3=60, T 1=50$, $T 2=70, S=230, F G=20$. We can see that TLR of both protocols increases as the tag density increases. The reason is that the reader has to identify more tags in the same interval. Compared with DFSA, GBDFSA has better performance. For example, when the number of tags in the second group $N 2$ is less than or equal to 80 tags, TLR of our protocol is nearly zero while that of DFSA is 25 percent.

In the Fig. 5 (b), we analyze the relationship between TLR and the tag sojourn time $S$ by changing $S$ (corresponding to change of the tag moving speed or the reader coverage area length). Related parameters in the experi- 
ment are: $N 1=60, N 2=60, N 3=60, T 1=50, T 2=70$, $F G=20$. We can see that TLR of both protocols decreases as $S$ increases. The reason is that the reader has more time to identify tags as $S$ increases. Compared with DFSA, the proposed protocol has better performance. For example, when tag sojourn time $S$ is larger than or equals 200 slots, TLR of GBDFSA is nearly zero. However, only when $S$ is larger than 400 slots, TLR of DFSA protocol achieves zero.

In the Fig. 5 (c), we analyze the relationship between TLR and time interval $T 1$ under the condition that $T 1+T 2$ is a constant (e.g. 120), which can to some extent derive which protocol is sensitive to changing $T 1$ in the same time span. Related parameters in the experiment are: $N 1=$ $60, N 2=60, N 3=60, S=250, F G=20$. We can find that TLR of GBDFSA is always equal to zero as $T 1$ increases from 10 to 110 under the condition of $T 1+T 2=120$ whereas for DFSA, the opposite is the case.

In the Fig. 5 (d), we analyze the relationship between TLR and tag arrival rate by changing $T 1$. Related parameters in the experiment are: $N 1=60, N 2=60, N 3=60$, $T 2=70, S=230, F G=20$. From the figure, we can find that TLR of both protocols decreases as the tag arrival rate become slow. The reason is that the reader has more time to identify tags when the rate becomes slow. Compared with DFSA, GBDFSA has better performance. When $T 1$ is larger than or is equal to 20 slots, TLR of GBDFSA is nearly zero. However, when $T 1$ is larger than 110 slots, TLR of DFSA protocol may achieve zero.

In the Fig. 5 (e), we analyze the relationship between TLR and the period (or frequency) of tag grouping by changing $F G$. Related parameters in the experiment are: $N 1=$ $60, N 2=60, N 3=60, T 1=50, T 2=70, S=230$. We can find that TLR of GBDFSA increases as the frequency of tag grouping decreases (corresponding to increase of $F G$ ). The reason is that RLI problem may not be resolved when the frequency of tag grouping is low. For example, when $F G>120$, the three groups of tags in the Fig 3.1 are not grouped and thus TLR of GBDFSA significantly increases and is equal to that of DFSA. We can also know easily that if the frequency of tag grouping is too high, great TLR may occur. The reason is that the operation of the tag grouping also needs the time cost. So, we can derive that the frequency (period) of tag grouping has a strong influence on TLR.

In general, the simulation results demonstrate that compared with DFSA, GBDFSA can reduce TLR and improve system performance remarkably since GBDFSA can eliminates influence of new arrival tags on tag quantity estimation algorithm.

\section{Conclusions}

The paper offers a method of tag grouping in the mobile RFID systems where the period of tag grouping is adjustable and the implementation of tag grouping does not need complicated computation. On this basis, we present

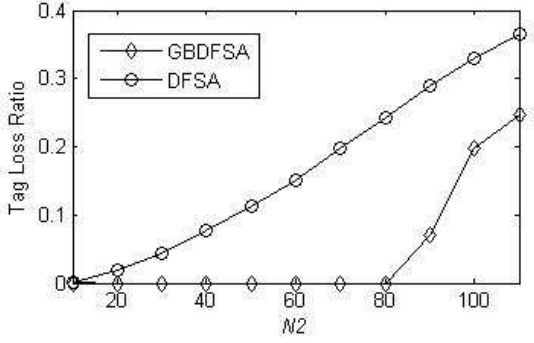

(a) TLR vs. the tag density

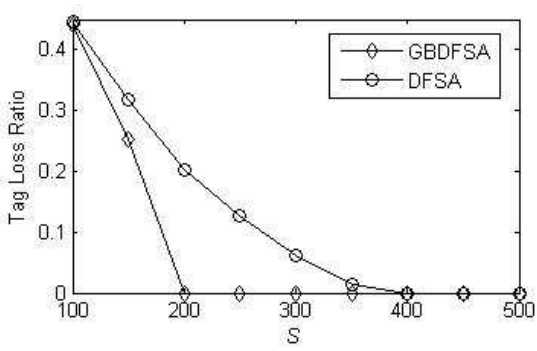

(b) TLR vs. the sojourn time

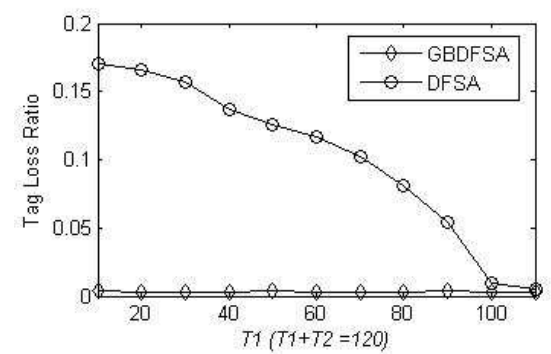

(c) TLR vs. T1 under the condition of $\mathrm{T} 1+\mathrm{T} 2=120$

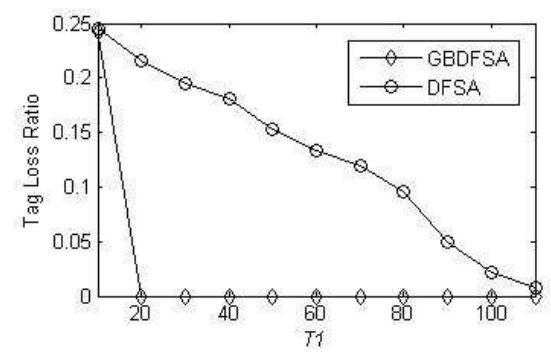

(d) TLR vs. tag arrival rate

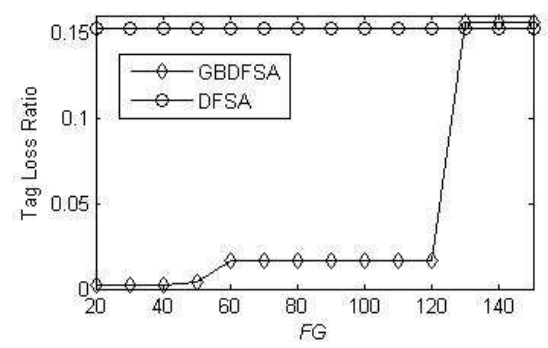

(e) TLR vs. the period of tag grouping

Figure 5 TRL of The proposed protocol and DFSA protocols. 
the GBDFSA protocol. In the protocol, the reader can identify tag groups (time groups) in their arrival order. Thus, our protocol can eliminate influence of new arrival tags on tag quantity estimation and tag identification based on GBDFSA protocol has FCFS feature. That is, our protocol can convert random access of tags into sequential access of tag groups. From the results presented in Section 3, it can be seen that GBDFSA can decrease TLR in the mobile systems significantly. Moreover, in order to evaluate the proposed protocol, we also offer a simplified mobile RFID experiment model, which can easily analyze the TLR of various protocols in the mobile RFID systems and avoid complicated mathematical computation of TLR. In future research, we will apply the idea of the paper to more dynamic framed slotted ALOHA protocols to decrease TLR.

\section{Acknowledgement}

This work is supported by the National Natural Science Foundation of China (NNSF) under Grant 60990320, 6099 0323, 61271090, and the National 863 Project of China under Grant 2012AA012305, and Sichuan Provincial Science and technology support Project under Grant 2012GZ 0101, and Chengdu Science and technology support Project under Grant 12DXYB347JH-002; and Science Foundation of Kunming University under Grant XJ11L001.

\section{References}

[1] Y.-C. Lee, Appl. Math. Inf. Sci. 6-2S, 425S-431S (2012).

[2] K. Finkenzeller, RFID Handbook: Radio-Frequency Identification Fundamentals and Applications, 2nd ed. New York: Wiley, (2003).

[3] Q. Lei, Appl. Math. Inf. Sci. textbf6, 453-457(2012).

[4] Y. Wang, H. Wang, Q. Zhang, Appl. Math. Inf. Sci. 6, 689695 (2012).

[5] L. Zhu and T.-S.P. Yum, IEEE Commun. Mag. 5, 214-221 (2011).

[6] D. K. Klair, K.-W. Chin, R. Raad, IEEE COMMUNICATIONS SURVEYS \& TUTORIALS, 12, 400-421 (2010).

[7] C.P. Wong and Q. Feng, IEEE Commun. Lett. 11, 946 - 948 (2007).

[8] X. Jia, Q. Feng and C. Ma, IEEE Commun. Lett. 14, 1014 1016 (2010).

[9] H. Vogt, Proceedings of International Conference on Pervasive Computing. Zurich, Switzerland, 98-113 (2002)

[10] W.-T. Chen, IEEE Trans. Autom. Sci. Eng. 1, 9-15 (2009).

[11] F. Schoute, IEEE Trans. Commun. 31, 565-568 (1983).

[12] K.C. Shin, S.B. Park and G.S. Jo, Sensors 9, 845-858 (2009).

[13] H. Wu, Y. Zeng, IEEE TRANSACTIONS ON AUTOMATION SCIENCE AND ENGINEERING, 7, 963-969 (2010).

[14] L. Xie, B. Sheng, C.C. Tan, H. Han, Q. Li, D. Chen, Proc. of IEEE International Conference INFOCOM, 15-19 (2010).

[15] J. Vales-Alonso, M.V. Bueno-Delgado, E. Egea-Lpez, J.J. Alcaraz, J.M. Prez-Manogil, Sensors 11, 2946-2960 (2011).

[16] J.J. Alcaraz, E. Egea-Lpez, J. Vales-Alonso and J. GarcłaHaro, Computer Networks 55, 74-83 (2011).
[17] J. Vales-Alonso, M.V. Bueno-Delgado, E. Egea-Lpez, J.J. Alcaraz-Espłn, J. Garcła-Haro, Proc. of 5th European Workshop on RFID Systems and Technologies, 16-17 (2009).

[18] V. Sarangan, M.R. Devarapalli, S. Radhakrishnan, Computer Networks 52, 1058-1073 (2008).

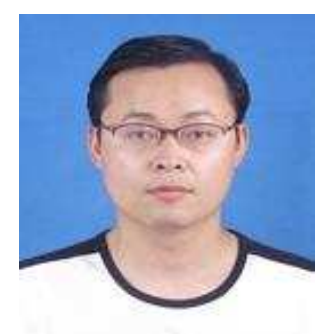

Xiaowu Li received the M.S. degrees in Computer Application Technology from Kunming University of Science and Technology, China, in 2007. Now he is PhD student in Southwest Jiaotong University, Chendu, China. His research interests include RFID technology, embedded system, wireless communications

and antennas etc.

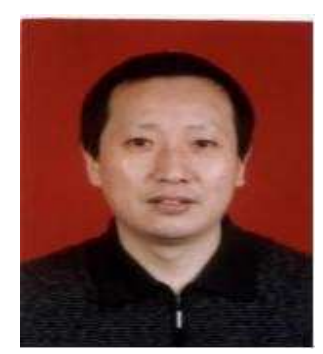

Quanyuan Feng is a professor and the head of institute of microelectronics of southwest Jiaotong University, China. He has been honored as "the excellent expert" and "the leader of science and technology" of Sichuan province because of his outstanding contribution. His research interests include RF and microwave devices, integrated circuits design, RFID technology, embedded system, wireless communications, antennas and propagation, microwave and millimeter wave technology, smart information processing, electromagnetic compatibility and environmental electromagnetics etc. 